\title{
CARATERIZAÇÃO DO DOENTE COM ENFARTE AGUDO DO MIOCÁRDIO
}

\author{
CHARACTERIZATION OF THE PATIENT WITH ACUTE MYOCARDIAL INFARCTION \\ CARACTERIZACIÓN DEL PACIENTE CON INFARTO AGUDO DE MIOCARDIO
}

Dora Fonseca Saraiva (dorasaraiva@ipg.pt)*

\begin{abstract}
RESUMO
A doença coronária tem enorme impacto na saúde pública, pelo que se torna fundamental a sua análise e caraterização. O estudo tem como objetivo caraterizar o doente com enfarte agudo do miocárdio no Centro Hospitalar Cova da Beira, quanto a dados demográficos, situação clínica, fatores de risco e intervenção/abordagem terapêutica e identificar áreas de potencial melhoria no atendimento destes doentes. Trata-se de um estudo quantitativo, descritivo, de caráter transversal e retrospetivo. A colheita de dados decorreu no último trimestre de 2011 e incidiu nos processos clínicos dos doentes com diagnóstico de EAM no ano de 2010. Os dados foram analisados com base na estatística descritiva e inferencial. De um total de 174 doentes, salienta-se uma amostra constituída maioritariamente homens com idade média de 73 anos. Entre os fatores de risco destaca-se a presença de hipertensão arterial seguida de dislipidémia e diabetes. O tempo médio dor-serviço de urgência foi 177 minutos e o tempo médio para a realização do $1^{\circ}$ electrocardiograma nos doentes triados com dor torácica foi 10.21 minutos. A fibrinólise foi realizada em 73.5\% dos doentes com um tempo porta-agulha médio de 56 minutos. A maioria dos doentes realizou coronariografia e $64.0 \%$ foram submetidos a angioplastia coronária. A mortalidade intra-hospitalar foi de 12.6\%. A análise dos resultados permite identificar diversos pontos que merecem atenção e que devem constituir objeto de futuras intervenções, de forma a garantir um melhor tratamento e prognóstico destes doentes. Destaca-se a necessidade de promover a admissão do doente com EAM através da via verde coronária e a sua acessibilidade às técnicas de intervenção coronária percutânea, dando cumprimento às recomendações nacionais e internacionais que evidenciam um claro impacto a curto, médio e longo prazo.
\end{abstract}

Palavras-chave: enfarte do miocárdio, doenças cardiovasculares, fibrinólise, angioplastia.

\begin{abstract}
Coronary heart disease has an enormous impact on public health, and, as such, its analysis and characterisation is pivotal. The study aims to characterise the patient with acute myocardial infarction at the Hospital Cova da Beira, as to the demographics, clinical condition, risk factors and intervention/therapeutic approach and to identify areas for potential improvement within the care provided to these patients. This is a quantitative, descriptive, cross-sectional and retrospective study. Data collection occurred in the last quarter of 2011 and focused on the medical records of patients diagnosed with AMI in 2010. The data were analysed by resorting to descriptive and inferential statistics. From the results, we point out a sample of 174 patients, mostly men with an average of 73 years of age. Among the risk factors, we highlight the presence of hypertension followed by dyslipidemia and diabetes. The average pain-emergency service time was 177 minutes and the average time until the performance of the
\end{abstract}


1st electrocardiogram in patients screened with chest pain was 10.21 minutes. Fibrinolysis was performed in 73.5\% of patients with an average needle holder time of 56 minutes. Most patients underwent coronary angiography and $64.0 \%$ were submitted to coronary angioplasty. Intra-hospital mortality was $12.6 \%$. The analysis of the results allows identifying several points worthy of attention and which should be object of future interventions, in order to ensure an improved treatment and prognosis for these patients. We emphasise the need to promote the admission of patients with AMl through the coronary "greenway" and their accessibility to percutaneous coronary intervention techniques, thus fulfilling the national and international recommendations which manifest a clear short, medium and long-term impact.

Keywords: myocardial infarction, cardiovascular diseases, fibrinolysis, angioplasty.

\section{RESUMEN}

Las enfermedades cardiovasculares, en concreto aquellas que afectan a las arterias coronarias, tienen un gran impacto sobre la salud pública, por lo que es fundamental su análisis e interpretación. El presente estudio tiene como objetivo la caracterización de los pacientes con infarto agudo de miocardio del Centro Hospitalar Cova da Beira, relacionando sus datos demográficos, situación clínica, factores de riesgo, enfoque terapéutico, identificando así, las áreas de potencial mejora en su atendimiento. Se trata de un estudio cuantitativo, descriptivo, transversal y retrospectivo. La información fue recopilada a través de los registros informáticos y los datos fueron analizados utilizando estadística descriptiva e inferencial. La recogida de datos tuvo lugar durante el último trimestre de 2011, incidiendo en las historias clínicas de los pacientes diagnosticados de IAM en el año 2010. De una muestra de 174 pacientes, varones en su mayoría y con un promedio de edad de 73 años, cabe destacar que el principal factor de riesgo fue la hipertensión arterial seguida de dislipidemia y diabetes. La duración media del dolor torácico en el servicio de urgencias fue de 177 minutos y el tiempo transcurrido desde el triaje hasta la realización del primer electrocardiograma fue de 10.21 minutos. La fibrinólisis fue realizada en el 73.5 \% de los pacientes con infarto en un promedio de tiempo de 56 minutos. La mayoría de estos pacientes realizo coronariografía y 64.0\% de estos fueron sometidos a angioplastia coronaria. La mortalidad intrahospitalaria fue del $12.6 \%$. El análisis de los resultados identifica varios puntos que merecen atención y deben ser objeto de intervenciones futuras, para garantizar un mejor tratamiento y pronóstico de este tipo de pacientes. Es necesario promover la admisión del paciente con IAM como prioridad I (vía verde coronaria en Portugal) y su rápida accesibilidad a medios de intervención coronaria percutánea primaria, cumpliendo con las recomendaciones nacionales e internacionales que evidencian un claro impacto a corto, medio y largo plazo.

Palabras-llave: infarto de miocardio, enfermedades cardiovasculares, fibrinólisis, angioplastia coronaria.

\footnotetext{
${ }^{*}$ Centro Hospitalar Cova da Beira (enfermeira com especialidade na área médico-
} cirúrgica) Mestre (Mestrado em Gestão de Unidades de Saúde)

Submitted: 21st March 2015

Accepted: 20th December 2015 


\section{Egitania \\ s c i e $\Omega$ c i a}

\section{INTRODUÇÃO}

As doenças cardiovasculares são a principal causa de morte a nível mundial (Perdigão et al, 2010). Na Europa, estas doenças são responsáveis por 23.0\% da morbilidade e cerca de metade de todas as mortes, causando, só na União Europeia, mais de 2 milhões de mortes anualmente (Patrão, 2009). Embora o seu peso relativo tenha sofrido um decréscimo gradual, a taxa de mortalidade por doença cardiovascular permanece inaceitavelmente elevada (INE, 2007).

Neste âmbito, insere-se a problemática do enfarte agudo do miocárdio (EAM) que, em Portugal regista uma taxa de mortalidade de 11,4\% num total de 12 mil enfartes diagnosticados anualmente (Aguiar citado por Cardoso, 2009). Para além de constituir uma fonte muito significativa de morbilidade e mortalidade, o EAM revela-se uma importante causa de recurso aos cuidados médicos de emergência e hospitalização e uma sobrecarga económica (Yavuz, 2008; Kolansky, 2009).

Considerando que um melhor conhecimento da realidade de cada país em geral, e de cada região em particular, poderá permitir aos agentes de saúde canalizar recursos, gerir o risco cardiovascular e definir estratégias de prevenção e tratamento adequados, surge este estudo com o objetivo de caraterizar o doente com EAM no Centro Hospitalar Cova da Beira (CHCB), quanto a dados demográficos, situação clínica, fatores de risco e intervenção/abordagem terapêutica e identificar áreas de potencial melhoria no atendimento destes doentes.

\section{QUADRO TEÓRICO}

No contexto das mortes por doença cardiovascular, 23.0\% são devidas a doença cardíaca isquémica, com destaque evidente para as Síndromes Coronárias Agudas (SCA), que compreendem um grupo de condições clínicas que resultam da doença aterotrombótica coronária em fase instável (Coordenação Nacional para as Doenças Cardiovasculares, 2007), incluindo a Angina Instável (AI), EAM com supra de ST (EAMCSST) e o EAM sem supra de ST (EAMSSST) (Teixeira 2009). Para este estudo foram apenas consideradas as 2 últimas entidades clínicas.

Na génese destes fenómenos estão os fatores de risco de doença cardiovascular aterosclerótica como condições que contribuem para o seu desenvolvimento. Estes fatores podem ser modificáveis quando numa perspetiva de prevenção podem ser corrigidos e, não modificáveis, quando não são passíveis de qualquer intervenção. Nos primeiros incluem-se: o tabaco, a dislipidémia, a hipertensão arterial (HTA), a diabetes, a obesidade, os fatores dietéticos, os fatores trombogénicos, a falta de exercício físico e o consumo excessivo de álcool; e nos segundos considera-se a história pessoal e familiar de doença cardiovascular, a idade e o género (Perdigão, 2011). 


\section{Egitania}

$s$ c i e $\Omega$ c i a

Uma vez diagnosticado o EAM requer intervenção médica urgente, já que "tempo é miocárdio!". As sociedades científicas têm vindo a propor guias clínicos e protocolos de atuação baseados na melhor evidência científica (Patrão, 2009). A atenção imediata a estes doentes tem uma relação direta com a probabilidade de sobrevivência. Estudos epidemiológicos demonstram uma taxa de mortalidade geral de 30.0\%, sendo que metade dos óbitos ocorre nas duas primeiras horas do evento. Acresce salientar que 14.0\% destes óbitos ocorrem antes do atendimento hospitalar (Teixeira, 2009).

O objetivo da intervenção é restaurar o fluxo coronário tão rapidamente quanto possível e salvar o dano miocárdio, uma vez que o aumento da morbilidade e mortalidade está associado ao aumento do tempo de início dos sintomas até ao tratamento (Teixeira, 2009). A reperfusão precoce nos doentes com EAM seja por terapia trombolítica e/ou por angioplastia coronária transluminal percutânea (ACPT) primária, melhoram a função ventricular esquerda e reduzem as complicações e a mortalidade (Teixeira, 2009). Estas intervenções constituem o meio mais eficaz para restaurar o equilibrio entre o aporte e a necessidade de oxigénio no miocárdio especialmente nos casos de oclusão trombótica persistente com zonas de necrose significativas já instaladas, sobretudo quando instituídas na primeira hora após o início dos sintomas (golden hour) (Pinto, 2010).

Apesar dos evidentes avanços tecnológicos e na área da terapêutica que se têm desenvolvido nas últimas décadas para a reperfusão miocárdica, vários fatores impedem o uso dessas terapias num número significativo de doentes. Estudos clínicos apontam que a demora préhospitalar tem sido o maior obstáculo e importante preditor de morbilidade e mortalidade (Teixeira 2009).

A melhoria das acessibilidades dos doentes na fase aguda das doenças cardiovasculares aos cuidados médicos mais adequados de diagnóstico e tratamento poderá conduzir a ganhos de saúde traduzidos na redução da morbilidade e da mortalidade hospitalar e global por EAM.

Neste contexto, as vias verdes enquanto estratégias organizadas para a abordagem, encaminhamento e tratamento mais adequado, planeado e expedito, nas fases pré, intra e interhospitalares, de situações clínicas mais frequentes e/ou graves devem ser especialmente valorizadas. Tal é o caso da via verde coronária que deve ser ativada em situações de EAM de forma a otimizar as acessibilidades e possibilitar os tratamentos mais eficazes a estes doentes (Coordenação Nacional para as Doenças Cardiovasculares, 2007).

\section{METODOLOGIA}

O estudo realizado é descritivo e quantitativo. Quanto ao período de referência, o estudo é transversal (uma vez que a recolha de dados se processou num determinado momento: último trimestre de 2011) e retrospetivo (pois os dados referem-se a uma situação clínica que ocorreu no passado: ano de 2010) (Freixo, 2009). 
A população diz respeito aos doentes com EAM do CHCB e a amostra não-probabilística por conveniência foi constituída por todos os doentes com diagnóstico confirmado de EAM do CHCB durante o ano de 2010, num total de 174. Foram consideradas variáveis demográficas (género e idade), variáveis clínicas (caraterização do episódio agudo, diagnóstico e perfil de risco) e variáveis relativas à abordagem/intervenção (tempo dor-serviço de urgência, tempo $1^{\circ}$ eletrocardiograma (ECG), reperfusão: fibrinólise e coronariografia, tempo porta-agulha e tempo porta-balão, destino e mortalidade intra-hospitalar.

Para a viabilização do estudo foi dado cumprimento a todos os preceitos éticos e formais inerentes à investigação em Saúde, nomeadamente ao parecer da Comissão de Ética do referido hospital. Os dados foram recolhidos através da análise dos processos clínicos de doentes com o diagnóstico de saída de EAM mediante consulta dos registos efetuados na base de dados do Alert巴 e SAM巴. Os doentes foram divididos em três grupos: não idosos [35-65[ anos, muito idosos ]85-100] anos e idosos [65-85] anos. A análise estatística foi realizada através do SPSS Statistics 17.0 para Windows®, utilizando-se para o efeito a estatística descritiva e inferencial. Uma vez que a amostra não apresenta distribuição normal utilizaram-se os testes não paramétricos Kruskal-Wallis e de Mann-Whitney. Considerou-se estatisticamente significativo um $p$-value bicaudal $<0.05$.

\section{RESULTADOS}

\section{Caraterização Demográfica}

A amostra em estudo foi constituída por 174 doentes com diagnóstico de EAM do CHCB, dos quais 63.8\% são homens e 36.2\% mulheres. A idade mínima foi 35 anos e a máxima 100 anos, sendo a média de 73 anos com um desvio padrão de 13 anos. Uma vez constituídos os grupos etários, 51.7\% situaram-se no grupo [65-85] anos, 27.6\% no grupo [35-65[ anos e 20.7\% no grupo ]85-100] anos. Os homens predominaram em todos os grupos etários com exceção do último onde as mulheres prevalecem (66.7\% vs. 33.3\%) (tabela 1).

\begin{tabular}{c|r|r|r|r|r|r}
\hline \multirow{2}{*}{ Género } & \multicolumn{2}{|c|}{ Masculino } & \multicolumn{2}{c|}{ Feminino } & \multicolumn{2}{c}{ Total } \\
\cline { 2 - 7 } Grupo etário & \multicolumn{1}{c|}{$\mathbf{n}$} & \multicolumn{1}{c|}{$\%$} & \multicolumn{1}{c|}{$\mathbf{n}$} & \multicolumn{1}{c}{$\%$} & \multicolumn{1}{c}{$\mathbf{n}$} & \\
\hline$[35-65[$ anos & 40 & 83.3 & 8 & 16.7 & 48 & 100.00 \\
{$[65-85]$ anos } & 59 & 65.6 & 31 & 34.4 & 90 & 100.00 \\
]85-100] anos & 12 & 33.3 & 24 & 66.7 & 36 & 100.00 \\
\hline Total & 111 & 63,8 & 63 & 36.2 & 174 & 100.00 \\
\hline
\end{tabular}

Tabela 1 - Distribuição da amostra tendo em conta o género e os grupos etários. 


\section{CARATERIZAÇÃO CLÍNICA}

Os doentes que integraram o estudo foram triados através do Sistema de Triagem de Manchester (STM), que determina o estabelecimento de prioridades de atendimento e define o tempo máximo que cada doente pode esperar por assistência diferenciada. Assim, 54.6\% foram triados com cor Amarela (situação urgente - tempo de espera para avaliação médica 1 hora), 34.5\% com Laranja (situação muito urgente - tempo de espera 10 minutos), e apenas 1.1\% com Vermelho (situação emergente - tempo de espera para avaliação médica O minutos). Nesta sequência, os descritores mais utilizados foram dor torácica com 44.8\%, indisposição no adulto com 19.5\% e dispneia com 17.8\%. A admissão através da via verde coronária aconteceu em 10.3\% da amostra.

No que concerne ao diagnóstico, 55.7\% apresentaram EAM não especificado, 24.7\% EAMSSST e 19.6\% EAMCSST. Relativamente ao território afetado, 16.3\% apresentaram enfarte inferior, $14.2 \%$ enfarte anterior, sendo que $21.1 \%$ tiveram mais que uma zona com isquémia/necrose e em 48.4\% o local não foi especificado. Quanto à caraterização da dor torácica verificou-se que 36.2\% não apresentaram dor, 30.5\% referiram dor torácica sem irradiação, 13.6\% dor torácica com irradiação para o membro superior esquerdo e 8.8\% do dor torácica com irradiação para a mandỉbula. Tendo em conta a sintomatologia atípica, 29.9\% apresentaram sintomas gástricos (náuseas/vómitos), 28.2\% sintomas respiratórios (dispneia/cansaço), 14.4\% sudorese e 13.2\% alterações da consciência (síncope).

No que diz respeito ao perfil de risco, constatou-se que 19.0\% não tinha qualquer fator de risco identificado, 35.9\% apresentavam 2 fatores de risco em simultâneo, 27.9\% 1 fator de risco e 17.2\% 3 fatores de risco. Especificando os fatores de risco presentes, 60.9\% apresentavam HTA, 41.4\% dislipidémia, 36.2\% diabetes e 15.5\% eram fumadores (tabela 2).

\begin{tabular}{c|r|r}
\hline Fatores de Risco & n & \multicolumn{2}{l}{$\%$} \\
\hline HTA & 106 & 60.9 \\
Dislipidémia & 72 & 41.4 \\
Diabetes & 63 & 36.2 \\
Tabagismo & 27 & 15.5 \\
\hline
\end{tabular}

Tabela 2 - Fatores de risco presentes na amostra.

Relativamente aos antecedentes cardiovasculares, 18.4\% apresentavam EAM prévio, 10.3\% doença coronária diagnosticada, 6.3\% acidente vascular cerebral (AVC), 2.3\% doença vascular periférica e 3.4\% mais do que um destes eventos associados. 


\section{Egitania}

$s$ c i e $\Omega$ c i a

\section{CARATERIZAÇÃO DA ABORDAGEM/INTERVENÇÃO}

66.7\% dos doentes com EAM recorreram ao Serviço de Urgência com dor torácica com mais de $2 \mathrm{~h}$ de evolução e o $1^{\circ}$ ECGfoi realizado com um tempo médio de 20,34min (mínimo de 1,00min e o máximo de 91,00min), valor que se reduz significativamente para 10,21 min se considerarmos apenas os indivíduos triados com descritor dor torácica.

A fibrinólise foi realizada em 73.5\% dos doentes com EAMCSST com um tempo porta-agulha médio de 56,00min (mínimo 4,00 min e máximo 161,00 min).

A coronariografia foi realizada em $67.1 \%$ dos doentes com EAM e destes 64.0\% foram submetidos a ACPT. Salienta-se que em $74.5 \%$ dos doentes o tempo porta-balão foi superior a 24h. Considerando o destino do doente, 68.0\% foram internados na Unidade de Cuidados Intensivos, 29.7\% no serviço de Cardiologia e 2.3\% foram transferidos para outra instituição. A mortalidade intra-hospitalar foi de 12.6\%.

\section{RELAÇÃO ENTRE AS VARIÁVEIS}

Analisando a relação das variáveis demográficas com as restantes variáveis, salientam-se os resultados estatisticamente significativos:

- Os indivíduos do grupo etário [35-65[ anos (não idosos) têm maior número de fatores de risco quando comparados com os restantes grupos ( $p$ value do Teste Kruskal-Wallis = 0.002);

- Os indivíduos do grupo etário ]85-100] anos (muito idosos) têm maior número de antecedentes cardiovasculares quando comparados com os restantes grupos ( $p$ value do Teste Kruskal-Wallis = 0.001);

- As mulheres recorrem mais tardiamente ao Serviço de Urgência quando comparadas com os homens ( $p$ value do Teste Mann-Whitney= 0.002);

- Os homens são mais sujeitos a fibrinólise quando comparados com as mulheres ( $p$ value do Teste Mann-Whitney=0.012).

- Os indivíduos do grupo etário [35-65[ anos (não idosos) são mais sujeitos a fibrinólise quando comparados com os restantes grupos ( $p$ value do Teste Kruskal-Wallis =0.000).

- Os indivíduos do grupo etário [35-65[ anos (não idosos) são sujeitos mais frequentemente a intervenção coronária percutânea (ICP) e a angioplastia quando comparados aos restantes grupos ( $p$ value do Kruskal-Wallis = 0.000 e 0.002 respetivamente);

- Os homens são sujeitos mais frequentemente a angioplastia quando comparados com as mulheres ( $p$ value do Teste Mann-Whitney $=0.011$ ). 


\section{Egitania}

s c i e $\Omega$ c i a

- A mortalidade intra-hospitalar é mais elevada nos indivíduos do grupo etário ]85-100] anos (muito idosos) ( $p$ value do Kruskal-Wallis = 0.030).

\section{DISCUSSÃO}

O Registo Nacional de SCA publicado por Santos et al (2009) é o maior e mais longo registo realizado em Portugal, tendo recolhido informação de 22482 doentes, internados em 45 hospitais das várias regiões do país, durante um período de sete anos. A população incluída no registo apresentava uma idade média inferior à obtida no estudo (66 anos), com um predomínio igualmente dos homens. Corroborando os resultados obtidos, no estudo realizado por Costa (2007), o SCA aconteceu maioritariamente entre homens até aos 74 anos, com as mulheres a predominar apenas na faixa etária acima de 75 anos.

A admissão pela via verde coronária ocorreu em 10.3\% dos casos. No estudo nacional (referido anteriormente) foi possível concluir que a admissão destes doentes através da via verde a nível nacional era de apenas 2.1\%. (Santos et al, 2009). A ativação desta via constitui o meio preferencial para aumentar a acessibilidade e eficácia da terapêutica de reperfusão do doente com EAM. Apesar do valor encontrado no presente estudo ser superior ao referido no estudo nacional, representa uma falha do sistema de saúde que urge otimizar, devendo ser alvo de reflexão e melhorias futuras.

No que diz respeito à triagem realizada através do STM, salienta-se que apenas 1.1\% dos doentes foram triados como emergentes e que o descritor dor torácica esteve presente em apenas 44.8\% da amostra. A percentagem de casos de EAM triados com sintomas atípicos é superior a um terço, tal como referido pela European Society of Cardiology (2012).

Um estudo realizado no mesmo hospital por Patrão (2009) durante os primeiros três meses de 2008 registou 755 episódios de dor torácica de prioridade emergente, muito urgente ou urgente referente a utentes com mais de 17 anos, dos quais 83.4\% foram triados como dor torácica urgente. No entanto, o fluxograma da maior parte dos doentes com EAM não foi dor torácica.

Ratificando o presente estudo, Teixeira (2009) refere que 68.2\% dos doentes com EAM referem como principal sintoma dor torácica e que cerca de metade (47.0\%) apresentaram sintomas adicionais (náuseas, vómitos, sudorese e dispneia).

Brieger et al (2004) citados por Pinto (2010) estudaram os sintomas predominantes em casos de SCA incluindo dor torácica (apresentação típica) ou não (atípica). De 20881 doentes, 8.4\% não se apresentaram com dor torácica, sendo a apresentação dominante destes doentes a dispneia (49.3\%), seguida da diaforese (26.2\%) e náuseas ou vómitos (19.1\%). Estes doentes eram significativamente mais velhos, do género feminino e apresentavam história de hipertensão, diabetes e insuficiência cardíaca. Tiveram menor probabilidade de ser tratados com terapêuticas efetivas e experimentaram morbilidade e mortalidade intra-hospitalar significativamente mais elevadas do que os que se apresentaram com dor torácica típica. 


\section{Eqitania}

$s$ c i e $\Omega$ c i a

Nesta perspetiva, a evidência científica comprova que a apresentação clínica e a queixa mais valorizada pelo doente são determinantes para todo o processo de triagem. A forma de apresentação nos casos atípicos é muito variada dificultando a identificação de um padrão que justifique a redução do limiar de suspeição, concluindo-se que dificulta o diagnóstico, atrasando ou inviabilizando a implementação de estratégias terapêuticas e de prevenção secundária com efetividade comprovada. Estes doentes apresentam um risco acrescido de atraso na procura de cuidados de saúde adequados, são submetidos a tratamentos hospitalares menos agressivos e sofrem maior mortalidade intra-hospitalar (Pinto, 2010).

Assim sendo, o desenvolvimento de protocolos que acelerem a avaliação dos doentes com queixas compatíveis com hipoperfusão do miocárdio, nomeadamente dor torácica, e também com outros sintomas associados a síndromes coronárias agudas, como dispneia, tem sido descrito como necessário e essencial para o funcionamento de um serviço de urgência (Patrão, 2009). Desta forma, torna-se pertinente a aplicação do protocolo de abordagem do doente com dor torácica também a outras situações, nomeadamente aos doentes cujo fluxograma é a dispneia.

Relativamente ao diagnóstico instituído, verificou-se neste estudo que 55.7\% dos doentes apresentaram EAM não especificado, 24.7\% EAMSSST e 19.6\% EAMCSST. Estes dados são congruentes com o estudo de Teixeira (2009), Lemos et al (2010) e de Pinto (2010). No entanto se observarmos os resultados do registo nacional da SPC publicado por Santos et al (2009) o tipo de SCA distribuiu-se da seguinte forma: 45.4\% com EAMCSST, 41.4\% com EAMSSST e 13.1\% com Al, verificando-se assim maior prevalência dos primeiros.

No que refere à demora pré-hospitalar, definida como o tempo decorrido entre o início dos sintomas e a admissão, 66.7\% dos indivíduos recorreram ao Serviço de Urgência com dor torácica com mais de $2 \mathrm{~h}$ de evolução e os restantes com menos de $2 \mathrm{~h}$, sendo que as mulheres recorrem mais tardiamente a este serviço quando comparadas com os homens ( $p$ value do Teste Mann-Whitney = 0.002). Por sua vez, na investigação lavada a cabo por Patrão (2009), o tempo médio entre o início da dor e a decisão de procurar atendimento médico foi 42.5min (15-187.5min) e o tempo médio entre o início da dor e a chegada ao serviço de emergência foi $191 \mathrm{~min}$ (25-374.25min).

O estudo conduzido por Teixeira (2009) refere que os principais fatores associados com a demora na procura de assistência são: idade avançada, género feminino, baixo nível educacional e status socio-económico, raça negra, condições crónicas como angina, diabetes, hipertensão, dislipidémia e tabagismo, início dos sintomas em casa, morar ou estar sozinho, interpretar incorretamente os sintomas, desconhecimento das terapêuticas de reperfusão, automedicação e longas distâncias.

O tempo porta-agulha foi em média de 56 min, sendo o tempo de espera preferencial de menos de 30 min, conforme recomendado pela European Society of Cardiology (2012) e pela Sociedade Portuguesa de Cardiologia (2012). A média do tempo porta-balão ficou muito aquém do preconizado pois na maioria dos casos foi superior a 24h. No hospital onde decorreu o 


\section{Egitania}

$s$ c i e $\Omega$ c i a

estudo, não existe serviço de hemodinâmica que permita realizar ACPT primária, sendo a fibrinólise o tratamento de eleição. A angiografia coronária ocorre num segundo plano, porém aquém das preferenciais primeiras 24 horas (European Society of Cardiology, 2012; Sociedade Portuguesa de Cardiologia, 2012).

Santos et al (2009) referem que para além da falta de redes de triagem e de referenciação préhospitalar, a forma como o doente chega à urgência do hospital e a própria triagem intrahospitalar poderão justificar parte do atraso verificado e devem ser alvo de reflexão e intervenções futuras.

Os resultados alcançados diferem das conclusões obtidas por Santos et al (2009, p.1477) no qual "o tempo porta-agulha foi de 177 min (90-418) e o tempo de demora entre a admissão e a administração da terapêutica de reperfusão foi de 60 min (30-119) para a fibrinólise (tempo porta-agulha) e de 96 min (52-175) para a angioplastia primária (tempo porta-balão) ( $\mathrm{p}<0.001$ para a diferença entre tempos). O tempo de demora entre o início dos sintomas e a administração da terapêutica de reperfusão foi 210 min (135-330) para a fibrinólise (tempo doragulha) e de 236 min (160-355) para a angioplastia primária (tempo dor-balão) ( $p<0.001$ para a diferença entre tempos)".

As orientações para o diagnóstico e tratamento da SCA e os vários documentos de consenso da European Society of Cardiology e da American Heart Association preconizam um tempo até à interpretação do $1^{\circ}$ ECG não superior a 10 min. Na admissão hospitalar o primeiro problema com que estes doentes se deparam é a necessidade de serem atendidos em tempo útil, ou seja, ser-Ihes atribuída uma prioridade de atendimento elevada, assegurando que um eletrocardiograma de 12 derivações é registado e interpretado num período máximo de 10min. No presente estudo e considerando o total da amostra o $1^{\circ}$ ECG foi realizado com um tempo médio de 20min (1-89min), valor que se reduz para 10.21 min no caso dos doentes triados com dor torácica. Dados semelhantes foram divulgados por Patrão (2009), o qual refere uma média de 20.34min (1-91 min). Os casos muito urgentes e emergentes foram alvo de um tratamento mais rápido que os urgentes mas ainda persiste uma demora excessiva em relação às recomendações, no que toca ao tempo do $1^{\circ}$ ECG em casos de dor torácica.

No que concerne ao território afetado pelo EAM, 48.4\% não foi especificado, 21.1\% tiveram mais que uma zona com isquémia, 16.3\% enfarte inferior e 14.2\% enfarte anterior. Em contrapartida, na análise de Santos et al (2009), os doentes com EAMCSST apresentaram como localização eletrocardiográfica mais frequente a inferior/lateral presente em $49.0 \%$ dos doentes, seguida da anterior em $45.0 \%$ e do bloqueio completo de ramo esquerdo de novo em $6.0 \%$.

Tendo em conta o perfil de risco, constatou-se que 60.9\% dos doentes tinham HTA, 41.4\% apresentavam dislipidémia, 36.2\% tinham diabetes e 15.5\% eram fumadores. Os dados são reflexo da prevalência dos fatores de risco clássicos na população portuguesa, conforme comprovam os estudos que se seguem. No estudo de Santos et al (2009), 85.0\% dos doentes tinha pelo menos um fator de risco cardiovascular clássico, sendo mais prevalente a HTA, 


\section{Egitania}

$s$ c i e $\Omega$ c i a

seguida da dislipidémia, da diabetes (4.5\% insulinotratada) e do tabagismo; na investigação de Lemos et al (2010) verificou-se a presença de sedentarismo em 86.8\%; sobrepeso e obesidade em 77.0\%; hipertensão arterial sistémica em 75.7\%; história familiar em 56.6\%; stress em 52.6\%; dislipidémia em 44.7\%; diabetes em 40.1\%; tabagismo em 39.5\% e ingestão de álcool em 14.4\%; e a análise de Teixeira (2009) revelou prevalência para hipertensão em 73.0\% e diabtes em 27.0\%. No estudo VALSIM o componente mais prevalente de síndrome metabólica foi a elevação da tensão arterial presente em 56.9\%, seguida da obesidade (46.3\%), hipertrigliceridémia (30.7\%) e elevação da glicémia em jejum (18.5\%). Com o aumento da idade, verificou-se a tendência para agregação adicional de mais fatores de risco, passando a elevação tensional e a obesidade a ser os elementos constantes nas variantes mais representadas (Fiúza et al, 2008).

Ainda a este respeito, verificou-se que em 19.0\% dos doentes não existia qualquer referência à identificação de fatores de riscos associados ao EAM, despertando a atenção para a necessidade de otimizar a aplicação efetiva das recomendações para identificação e registo dos fatores de risco cardiovascular.

Relativamente aos antecedentes cardiovasculares, 18.4\% tiveram EAM prévio, 10.3\% tinham doença coronária diagnosticada, 6.3\% AVC, 2.3\% doença vascular periférica e 3.4\% mais do que um evento associado. De forma similar, no estudo nacional em 44.0\% da população existia história prévia de doença cardiovascular, com destaque para a presença de angor e de antecedentes de EAM (Santos et al, 2009).

No presente estudo, foi efetuada terapêutica de reperfusão nos doentes com EAMCSST recorrendo-se preferencialmente numa $1^{\text {a }}$ fase à terapêutica fibrinolítica (73.5\%) e posteriormente a ICP (67.1\%). Na investigação de Santos et al (2009) apenas 17.8\% dos indivíduos foram sujeitos a fibrinólise e $66.7 \%$ a ICP. Cerca de um terço dos doentes não foram submetidos a nenhum procedimento de reperfusão, dos quais, em 55.0\% a apresentação tardia foi a principal contra-indicação para a fibrinólise (25.0\% apresentavam mais de 7h de evolução dos sintomas no momento da admissão, seguida da HTA não controlada em 3.9\% e da hemorragia recente em 2.0\%).

Estes resultados confrontam-se com a tendência crescente para um incremento na utilização da angioplastia primária em detrimento da fibrinólise (Santos et al, 2009), tornando-se, assim, objetivo principal da implementação da via verde coronária, o diagnóstico correto e expedito e o tratamento adequado dentro da janela terapêutica confirmadamente mais eficaz.

Em 64.0\% dos doentes submetidos a coronariografia foi realizada revascularização miocárdica com preferência óbvia pela ICP. Situação idêntica foi verificada por Santos et al (2009) onde esse valor se situou nos $62.5 \%$ do total da amostra. Neste contexto, foi ainda possível confirmar que, os indivíduos do grupo etário [35-65[ anos são mais sujeitos a fibrinólise, a ICP e a angioplastia quando comparados com os restantes grupos.

A evidência científica comprova que o tratamento das SCA na população idosa deverá ser idêntico aos restantes grupos etários, porém diversos estudos internacionais têm igualmente 


\section{Egitania}

$s$ c i e $\Omega$ c i a

demonstrado abordagens terapêuticas mais conservadoras nos doentes idosos. Constituindo os idosos um grupo com risco elevado em termos de prognóstico, é também um grupo com risco acrescido de complicações associadas ao tratamento, o que condiciona fortemente a sua abordagem (Timóteo et al, 2011).

Em conformidade, Costa (2007) concluíu que os idosos muitas vezes recebem tratamentos menos agressivos ou deixam de receber tratamentos já consagrados, com comprovados benefícios, mesmo quando indicados pelas diretrizes vigentes. Vários são os motivos que podem explicar as possíveis causas para esse aparente desinteresse em relação ao tratamento optimizado dos idosos com SCA. Entre esses, a preocupação com os efeitos colaterais dos medicamentos utilizados, em decorrência das alterações metabólicas e comorbidades associadas ao envelhecimento e os riscos das terapêuticas invasivas, como maior frequência de hemorragia e desfechos adversos. $\bigcirc$ facto dos idosos muitas vezes terem um diagnóstico tardio, por apresentarem quadros atípicos quanto às manifestações clínicas e pelas patologias coexistentes poderem mascarar um diagnóstico imediato, pode privar os idosos dos benefícios de terapêuticas que tenham o fator tempo como limitante na sua aplicação, como é o caso dos trombolíticos.

Ratificando os resultados alcançados, nesta pesquisa o tratamento invasivo com uso de trombolíticos revelou diferença significativa com tendência linear de menor uso conforme o avançar das faixas etárias e em relação à angioplastia, os muito idosos fizeram menos angioplastias primárias. No estudo de Costa (2007, p. 62), "a idade muito avançada também foi fator para menor utilização de tratamento invasivo durante a hospitalização, com 80.0\% nos doentes menores de 65 anos e apenas 54.0\% nos idosos com mais 85 anos a realizar angioplastia".

No total de doentes incluídos neste estudo, a mortalidade intra-hospitalar média foi de $12.6 \%$, sendo que os mais idosos têm taxas de mortalidade mais altas. Este resultado é corroborado por toda a literatura revisada. Com efeito, o risco de mortalidade intra-hospitalar é 6.3 vezes superior nos doentes muito idosos comparativamente com a faixa etária entre os 45 e 64 anos. Nos idosos, a função renal e a função ventricular esquerda são os fatores preditores mais importantes de mortalidade a curto e médio prazo (Timóteo et al, 2011).

Similarmente, Feliciano et al (2005) comprovam que a idade constitui um fator independente predisponente de mortalidade intra-hospitalar (19.6\% no grupo dos mais idosos e de 4.5\% no grupo com idade inferior a 75 anos, $p<0.001$ ) diretamente influenciada pelo pesado perfil clínico e extensão da doença coronária observados nestes doentes de alto risco.

\section{CONCLUSÃO}

Os dados obtidos neste estudo podem influenciar o planeamento em saúde, como processo ativamente participado, identificando diversos pontos que despertam a atenção, ao mesmo tempo que clarifica as áreas prioritárias que devem constituir objeto de intervenções futuras. 


\section{Egitania}

$s$ c i e $\Omega$ c i a

Assim, no âmbito de uma visão estratégica para melhorar a prevenção cardiovascular, salientase uma alta prevalência de fatores de risco para enfarte na população em estudo, entre os quais a HTA, dislipidémia e diabetes. Como sugestão reforça-se a necessidade de implementação de medidas de intervenção primária, através de sessões de educação para a saúde com o intuito de consciencializar a comunidade para o problema em análise e agir sobre os fatores de risco modificáveis para doença cardiovascular (nomeadamente HTA, dislipidémia, diabetes e tabagismo). A respeito disso, reforça-se a aplicação alargada de escalas de risco (sistema SCORE) por todos os profissionais de saúde e a implementação das recomendações adequadas a cada nível de risco.

Merece igualmente destaque, a necessidade de melhoria das acessibilidades e o tratamento atempado do EAM, objetivado através da implementação eficaz da via verde coronária, uma vez que através dela é possível contribuir para a redução da mortalidade padronizada e da letalidade intra-hospitalar, do diagnóstico precoce e da utilização de terapêuticas farmacológicas de prevenção primária e secundária para as doenças cardiovasculares.

Assim, no sentido de optimizar a via verde coronária, o cidadão, como principal beneficiário da sua implementação, deverá ser informado dos sintomas e sinais de alerta das situações cardiovasculares agudas e da utilização preferencial do número nacional de emergência médica (112) nessas situações. Neste sentido, sugerem-se intervenções de educação e aconselhamento com o objetivo de reduzir a demora na procura de tratamento, melhorar o conhecimento sobre a natureza dos sintomas cardíacos e atitudes a serem tomadas em doentes com risco de eventos futuros.

No âmbito da abordagem/intervenção do EAM, os dados demonstram alguma discordância entre as recomendações existentes e a sua aplicação na prática clínica. Ao contrário do recomendado, a fibrinólise prevalece em detrimento da ACPT primária. Desta forma, considerase fulcral facilitar o acesso às técnicas de intervenção coronária percutânea, aumentando o número de doentes tratados com esta intervenção de forma a dar cumprimento às recomendações internacionais que evidenciam um claro impacto no prognóstico a curto, médio e longo prazo.

No presente estudo foi ainda possível comprovar que os idosos recebem menos tratamentos invasivos deixando de receber terapias legitimadas por ensaios clínicos randomizados e indicadas pelas diretrizes internacionais. Neste grupo de doentes de alto risco não existe perda dos benefícios expetáveis da angioplastia primária, apoiando-se a necessidade de uma estratégia agressiva neste grupo da população.

O presente estudo apresenta como principal limitação a impossibilidade de generalização dos resultados para a população em geral (número reduzido de elementos em estudo, métodos estatísticos não-paramétricos, espaço temporal apenas de 1 ano). Apesar disso, o estudo contribui para uma melhor compreensão da realidade do doente com EAM atendido no CHCB, possibilitando no futuro o desenvolvimento de estratégias que facilitem ações preventivas e 


\section{Egitania \\ s c i e $\Omega$ c i a}

intervenção precoce (descritas anteriormente) promovendo a melhoria contínua da qualidade dos cuidados prestados e consequentemente do prognóstico destes doentes.

\section{REFERÊNCIAS}

CARdoso, José (2009). A QUEM diz RESPEITO O COMBATE ÀS DOENÇAS CARDIOVASCULARES.. NOtíCIAS DO XXX CONGRESSO PORTUGUÊS DE Cardiologia da Sociedade Portuguesa de Cardiologia, P. 4-5.

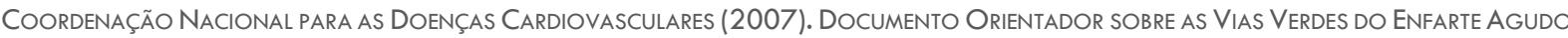
do Miocárdio e do Acidente Vascular Cerebral. lisboa: Alto Comissariado da Saúde.

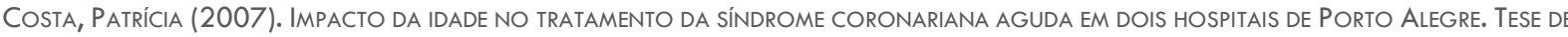
Doutorado em Clínica Médica e CiênCIas da Saúde. FaCuldade de Medicina da Pontifícia Universidade CatÓlica do Rio Grande do Sul. BRASIL: PORTO AlEGRE.

EUROPEAN SOCIETY OF CARDIOLOGY (2012). ESC GUIDELINES FOR THE MANAGEMENT OF ACUTE MYOCARDIAL INFARCTION IN PATIENTS PRESENTING With ST-SEgment eleVATION. Eur HeART J., 33, P. 2569-2619.

Feliciano, Joana [et al.] (2005). Angioplastia Directa como Terapêutica de Reperfusão no IdOSO aCima dos 75 AnOS. ReVista Portuguesa de CARDiologia, 24 (2), P. 205-214.

Freixo, MANuel (2009). Metodologia Científica. Fundamentos, métodos e téCniCAs. Lisboa: Instituto Piaget.

Fuiza, MANuela [et al.] (2008). Síndrome Metabólica em Portugal: PrevalênCia e ImplicaçÕes no RisCo Cardiovascular- Resultados do estudo VALSIM. ReVISTA PORTUguesa de CARDIOlOGIA, 27 (12), P. $1495-1529$.

Instituto Nacional de Estatística (2007). AnuÁrio Estatístico de Portugal. Lisboa: INE.

KOLANSKY, DANIEL (2009). ACUTE CORONARY SYNDROMES: MORBIDITY, MORTALITY, AND PHARMACOECONOMIC BURDEN. AM J MANAG CARE, 15 (2 SUPPL), P. S36-41.

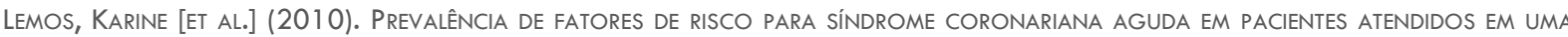
emergênCia. ReVISta Gaúcha Enfermagem, 31 (1), P. 129-135.

OMS (2009). Cardiovascular diseases. Genebra: World Health Organization.

PATRÃO, LUIS (2009). PROTOCOlO de ACTUAÇÃO NA dOR TORÁCICA - TEMPOS DE DEMORA INTRA-HOSPITALAR NOS CASOS DE DOR TORÁCICA E DE

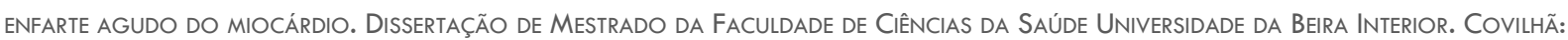
UBI.

Perdigão, Carlos (2011). Risco Cardiovascular Global. Revista Factores de Risco da Sociedade Portuguesa de Cardiologia, 20 (6), P.58-61.

Perdigão, Carlos [et al.] (2010). Risco Cardiovascular Global. ReVista Factores de Risco da Sociedade Portuguesa de Cardiologia, $17(4)$, P. 12-19.

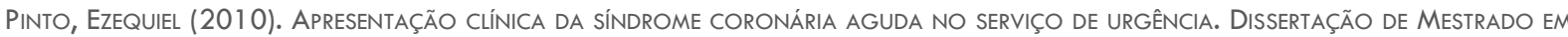
Saúde Pública. Faculdade de Medicina da Universidade do Porto. Porto: FMUP.

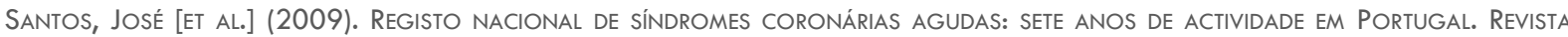
PORTUGUeSA DE CARDIOLOGIA, 28 (S.N.), P. 1465-1500.

Sociedade Portuguesa de CARdiOlogia (2012). EAM - STEMI. ReCOMENDAÇÕES PARA O TRATAMENTO dO ENFARTE AGUdO dO MIOCÁRDIO EM DOENTES COM ELEVAÇÃO PERSISTENTE do SEGMENTO ST. LISBOA: SOCIEDADE PORTUGUESA de CARDIOLOGIA. 


\section{Eqitania}

s c i e $\Omega$ c i a

TEIXEIRA, CÁtIA (2009). IMPACTO dO RECONHECIMENTO PRECOCE DOS SINAIS E DOS SINTOMAS DE SINDROME CORONARIANA AGUDA NO TEMPO DE procura por atendimento de emerGênCIA. Dissertação de Mestrado em CiênCias da Saúde: Cardiologia e Doenças Cardiovasculares. Universidade Federal do Rio Grande do Sul. Brasil: Porto Alegre.

TIMÓTEO, ANA [ET AL.] (201 1). IMPACTO DA IDADE NO TRATAMENTO E RESULTADOS APÓS ENFARTE AGUDO DO MIOCÁRDIO EM PARTICULAR NOS MUITO idosos. ReVISTA Portuguesa de CARDIOlogia, 30 (12), P.897-903.

YAVUZ, Senol (2008). Surgery as early revascularization after aCute myocardial infarction. The Anatolian Journal OF Cardiology, 8 (SUPPL 2), P. 84-92. 